\title{
INAUGURAL EDITORIAL NOTE
}

Welcome to volume one, issue one, of Journal of Computational Interdisciplinary Sciences (JCIS). Since its conception in summer of 2007, some members of the editorial board have worked hard to bring together financial and intellectual support in order to make it a new and real scientific endeavour. JCIS is the official journal of Pan-American Association of Computational Interdisciplinary Sciences (PACIS).

The PACIS is an autonomous association that aims to promote the understanding, development, scientific and education practice of interdisciplinary sciences where the computational science is explicitly one of the methodological components.

Objectives of PACIS include:

- To establish a new emergent interdisciplinary community on scientific issues involving applications of computational mathematical modeling, correlated analytical approaches and numerical techniques, computational simulations and analysis for more accurate descriptions, predictions, experimental observations and applications of the phenomenological processes in science and technology;

- The publication of this Journal of Computational Interdisciplinary Sciences (JCIS), for a main audience formed by: computing scientists, mathematicians, physicists, chemists, biologists, engineers, Earth and environmental scientists and others researches involved in specific applications of advanced computational methods, algorithms, techniques, simulations and high performance computing.

In particular, the primary objective of JCIS is to provide a single forum for the computational interdisciplinary area to strengthen links between basic and applied research, theoretical and experimental methods relating scientific computing and computational mathematics. Its goal is to bring together, in one periodical, significant papers of high quality on all aspects of interdisciplinary sciences when the scientific computing is one necessary branch.

Thus, the scope of this new international journal includes mainly advanced computational issues related to mathematics, physics, chemistry, biology and engineering of complex systems in numerical and natural experiments. Also applied computing in social and economic sciences and the other areas of science \& technology are considered by the editorial board. Then, it is of practical interest to publish papers on the application of computer simulation and other forms of computation to problems in various scientific disciplines. In this sense, the scope of JCIS is distinct from pure computer science, understand here as the mathematical study of computation, computers and information processing. Scientists and engineers, not graduate in computer science, having computing skills develop specific computer programs, application software that model systems being studied and run these programs with various sets of input parameters. Usually, these applied models require massive amounts of calculations and are often executed on high-performance computing (supercomputers and distributed platforms as clusters and grids). In this framework, the scientific computing approach is to gain understanding, mainly through the analysis, visualization and modeling implemented on and for computers.

This first issue of JCIS contains six original invited papers. The first contribution, in the area of computational mathematics, describes computational and mathematical methods based on the formal heuristic approach. The second contribution is on pattern formation from numerical experiments of typical interest in nonlinear phenomena studied in computational physics and chemistry. 
The third paper, related to computational physics, discusses the so-called stochastic touristic walk showing the algorithm and numerical results which have applications in several natural phenomena. The fourth paper, fitted in computational biology, is related to glucose transport modeling. The other two papers use advanced computational methods applied in remote sensing computational data analysis. Thus, the fifth and sixth papers address subjects that can be fitted in the interdisciplinary emergent area of applied computing in space and environmental sciences. All papers are developed by computational scientists, used here to describe someone skilled in scientific computing. This person is usually a scientist (physicist, biologist, etc), an applied mathematician, or an engineer who applies scientific computing in different ways to advance the state-of-the-art in their fundamental disciplines in physics, chemistry, biology and engineering. They can also be found in more intrinsically multidisciplinary areas where the scientific computing has increasingly impacted as medicine, economics, sociology and linguistic.

This publication seeks to bridge the artificial walls among many kinds of scientific disciplines that persist even when they have the scientific computing as a common explicit language. The focus of our journal is to explicit as much as possible the computational content of the research, usually hidden or neglected due to the weight of specific fundamental areas or editorial constraints in noncomputational disciplinary journals. In this sense, we take pride in introducing this new interdisciplinary publication and we are grateful for all of the help we have received from our relatives, colleagues, scientists, students, editors, publishers, sponsors and agencies.

\section{Reinaldo R. Rosa \\ General Secretary \\ PACIS}

Editor-in-Chief 